\title{
The Effect of Methylsulfonylmethane on Hair Growth Promotion of Magnesium Ascorbyl Phosphate for the Treatment of Alopecia
}

\author{
Srinivasan Shanmugam, Rengarajan BASKARAN, Santhoshkumar NAGAYYA-SRIRAman, \\ Chul-Soon YoNG, Han-Gon CHOI, Jong Soo Woo, and Bong-Kyu Yoo* \\ College of Pharmacy, Yeungnam University, Kyungsan 712-749, Republic of Korea
}

(Received April 10, 2009; Revised May 16, 2009; Accepted May 23, 2009)

\begin{abstract}
The purpose of this study was to evaluate the effect of methylsulfonylmethane (MSM) on hair growth promotion of magnesium ascorbyl phosphate (MAP) for the treatment of alopecia. Aqueous solutions of MAP $7.5 \%$ with or without MSM $1 \%, 5 \%$ or $10 \%$ were prepared and applied onto the depilated back skin of the male mice once a day for 20 days. The degree of hair growth was evaluated by visual scoring using hair growth quantification scale ( $0-5,0$ being initial state and 5 being complete hair growth). In vitro transdermal penetration and intradermal retention studies of MAP were performed with Franz diffusion cell using hairless mice skin. Hair growth in the group treated with the aqueous solution containing MAP 7.5\% and MSM 10\% was comparable to or better than the result in the group treated with minoxidil $5 \%$ solution. Hair growth promotion of MAP was dose-dependently increased by the presence of MSM used in combination with MAP $7.5 \%$ solution. The in vitro transdermal penetration of the MAP was decreased in proportion to the concentration of MSM. However, intradermal retention of MAP was profoundly and dose-proportionally increased as a function of MSM concentration, reaching $802 \mu \mathrm{g} / \mathrm{cm}^{2}$ in the presence of MSM $10 \%$ (200-fold increase). The effect of MSM on hair growth promotion of MAP was dose-proportional to the concentration of MSM due to the enhanced intradermal retention of MAP in the presence of MSM. Therefore, topical application of MAP together with MSM appears to be useful for the treatment of alopecia.
\end{abstract}

Keywords: Magnesium ascorbyl phosphate, Methylsulfonylmethane, Alopecia, Hair growth, Skin permeation/accumulation, Permeation enhancer

\section{INTRODUCTION}

Magnesium ascorbyl phosphate (MAP) (Fig. 1A), a stable phosphate derivative of L-ascorbic acid has been reported to stimulate growth of dermal papilla cells in vitro (Hata and Senoo, 1989; Takamizawa et al., 2004) and induce early conversion of telogen phase to anagen phase of hair cycle in experiment with mice (Sung et al., 2006). Furthermore, MAP treatment resulted in significant elongation of hair shafts in isolated hair follicles. Mechanism responsible for the growth stimulation of dermal papilla cells and elongation of hair shafts has been suggested that MAP induces proliferative and anti-apoptotic effects on dermal papilla cells (Sung et al., 2006). This finding provided a potential that MAP might be useful for hair growth

${ }^{*}$ Corresponding author

Tel: +82-53-810-2822 Fax: +82-53-810-4654

E-mail: byoo@ynu.ac.kr promotion in the treatment of alopecia. However, because of its hydrophilicity MAP has lower ability to penetrate the skin barrier stratum corneum to produce its therapeutic effect (Spiclin et al., 2002; Yoo et al., 2008).
A

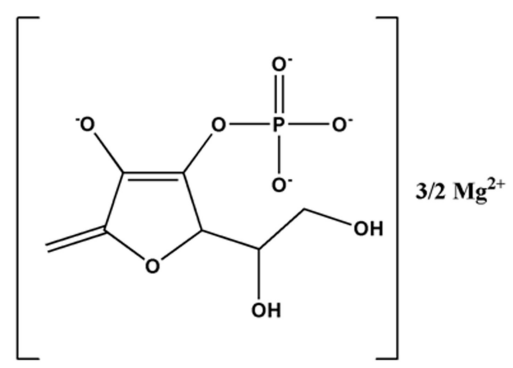

B

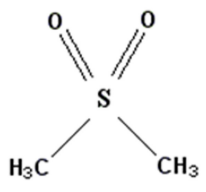

Fig. 1. The chemical structures of magnesium ascorbyl phosphate (MAP) and methylsulfonylmethane (MSM). (A) Magnesium ascorbyl phosphate (MAP). (B) Methylsulfonylmethane (MSM). 
In transdermal drug delivery arena, several approaches have been employed to lessen the barrier function of the stratum corneum for enhanced penetration of drug of choice, and one popular approach has been the use of trandermal permeation enhancers (Godwin and Michniak, 1999; Asbill and Michniak, 2000). These are chemical compounds that reversibly alter the barrier function of the skin and enhance penetration. Among various classes of permeation enhancers, those that enhance local skin-drug concentrations (intradermal) and do not produce significant transdermal enhancement (across the skin) are considered ideal permeation enhancers for topical delivery (Asbill and Michniak, 2000; Im et al., 2008). Myriad of researchers reported the possible mechanisms and uses of various penetration enhancers. However, despite major interest in this research arena, very few penetration enhancers have been made commercially available because of the limitations on selection based on their efficacy, lack of toxicity, and compatibility with other components of the transdermal system (Skelly et al., 1987). One of the earliest and widely investigated penetration enhancers is DMSO (Williams and Barry, 1992).

Methylsulfonylmethane (MSM), also known as dimethyl sulfone or methyl sulfone (Fig. 1B), is an organic sulfur-containing compound that occurs naturally in a variety of plants and animals including humans (Pearson et al., 1981; Usha and Naidu, 2004; Ameye and Chee, 2006). MSM is a normal oxidative metabolite product of dimethylsulfoxide (DMSO) and has been widely used as a dietary supplement for the treatment of several indications such as osteoarthritis, seasonal allergic rhinitis, interstitial cystitis, and snoring (Childs, 1994; Barrager et al., 2002; Blum and Blum, 2004; Kim et al., 2006). Like DMSO, MSM is an aprotic solvent that can dissolve wide range of solutes and is miscible with many solvents, and therefore could be easily incorporated into pharmaceutical formulations (Clark et al., 2008).

It is well known that much of the unique properties of DMSO are related to its characteristic polar O-S bond, which enables the compound to interact strongly with water by forming hydrogen bonds. This interaction with water is thought to be one of the key elements in the mechanism of action of DMSO as a penetration enhancer (Franz et al., 1995). Due to the structural similarity with DMSO, MSM is expected to act as a skin permeation enhancer for various medications by similar mechanisms (Engelen and Engelen, 2002). Structural similarity coupled with non-toxicity and safety (GRAS Notice Number: GRN 229) as an oral nutritional additive as well as unavailability of prior work, put forth MSM a rightful candidate to explore its permeation enhancing potential among many other permeation enhancer.

In this study, we have hypothesized that hair growth promotion effect of MAP can be improved by co-application with MSM because of its skin permeation enhancing effect. The purpose of this study was (1) to evaluate in vivo hair growth promotion effect of MAP (in the absence and presence of MSM) using alopecia model of C57BL/6N mice and (2) to substantiate the in vivo result with transdermal penetration and intradermal retention of MAP in vitro.

\section{MATERIALS AND METHODS}

\section{Materials}

Magnesium ascorbyl phosphate, methylsulfonylmethane, and methyl paraben were obtained from Wako Pure Chemical Industries (Osaka, Japan). Thioglycolic acid $80 \%$ cream (Niclean Cream ${ }^{\circledR}$, Ildong Pharmaceutical Inc., Korea) and minoxidil $5 \%$ solution (Minoxyl Solution ${ }^{\circledR}$, Hyundai Pharmaceutical Inc., Korea) were purchased from a local pharmacy. Sodium phosphate monobasic monohydrate and sodium phosphate dibasic heptahydrate were purchased from Sigma-Aldrich, Inc., (St. Louis, USA). Metaphosporic acid and trichloroacetic acid were purchased from Junsei Chemical Company Ltd., (Tokyo, Japan). Methanol used was HPLC grade and all other chemicals were of analytical grade and used without further purification.

\section{Preparation of MAP solutions}

MAP (7.5 g) was accurately weighed and dissolved into $100 \mathrm{ml}$ distilled water. Aliquots of this solution $(10 \mathrm{ml})$ were taken and added with 100,500 , or $1,000 \mathrm{mg}$ of MSM to make $1 \%, 5 \%$, or $10 \%$ MSM in the MAP $7.5 \%$ solution. The resultant aqueous solutions of MAP $7.5 \%$ alone or with MSM $1 \%, 5 \%$ or $10 \%$ were thoroughly mixed with magnetic bar and used for in vitro or in vivo studies thereafter. Compositions of aqueous solution of MAP containing various concentrations of MSM are shown in Table I.

Table I. Compositions of aqueous solution of MAP containing various concentrations of MSM

\begin{tabular}{lccc}
\hline & MAP & MSM & Solvent \\
\hline MAP 7.5\% only & $7.5 \%$ & - & Distilled water \\
MAP-MSM 1\% & $7.5 \%$ & $1 \%$ & Distilled water \\
MAP-MSM 5\% & $7.5 \%$ & $5 \%$ & Distilled water \\
MAP-MSM 10\% & $7.5 \%$ & $10 \%$ & Distilled water \\
\hline
\end{tabular}

MAP: magnesium ascorbyl phosphate, MSM: methylsulfonylmethane, MAP-MSM: MAP $7.5 \%$ in the presence of designated concentration of MSM. 


\section{HPLC analysis}

The concentration of MAP in transdermal penetration and intradermal retention studies, was assayed by HPLC system (Shimadzu, Japan) equipped with Class VP computer software, LC 10 AD VP pump, and SPD 10A UV-VIS detector at $240 \mathrm{~nm}$ using methyl paraben as internal standard. Column used was Inertsil CN-3 $(4.6 \times 250 \mathrm{~mm}$, GL Science Inc, Japan) and mobile phase consisted of a mixture of $0.02 \mathrm{M}$ phosphate buffer and methanol (60:40, $\mathrm{v} / \mathrm{v}$ ) adjusted to $\mathrm{pH} 2.3$ with phosphoric acid. Flow rate was $0.8 \mathrm{ml} / \mathrm{min}$ and the injection volume of the sample was $20 \mu \mathrm{l}$. The validation of the HPLC assay was performed by repeating five times a day for five consecutive days for MAP concentration range of $50 \mathrm{ng} / \mathrm{ml}-100 \mu \mathrm{g} / \mathrm{ml}$.

\section{Animals}

Animals care and procedures were conducted according to the guidelines for animal use in toxicology (Society of Toxicology USP 1989) and the study protocol was approved by the Animal Care and Use Committee, College of Pharmacy, Yeungnam University. Six-week-old male mice (C57BL/6N) and six-week-old male hairless mice were obtained from Orient Bio (Seoul, Korea) and housed in groups not exceeding six per cage and maintained under standard conditions. The acclimation period was two weeks before the experimental procedure with a dark/light cycle of $12 \mathrm{~h} / 12 \mathrm{~h}$ at the temperature of $23 \pm 2^{\circ} \mathrm{C}$. Food and tap water were available ad libitum during the acclimation period.

\section{Evaluation of hair growth promotion using alopecia model in mice}

The hair on the back of male C57BL/6N mice was removed by electric clipper and depilated using thioglycolic acid $80 \%$ cream (Niclean cream $^{\circledR}$, Ildong Pharmaceutical Inc., Korea) one day before applying the samples. About $0.5 \mathrm{~g}$ of the cream was applied on the hair-clipped back of the mice and allowed for $2 \mathrm{~min}$. The cream and hair were wiped off using cotton for depilation, and the back skin was rinsed with plenty of distilled water to remove remaining thioglycolic acid. The mice without significant wound on the back were selected, randomly grouped, and used for hair growth study. Freshly prepared aqueous solution (0.2 ml) of MAP $7.5 \%$ alone or with MSM $1 \%, 5 \%$ or $10 \%$ was applied onto the depilated back of the mice $(n=5)$ and allowed to dry. The sample application was done once a day for twenty days. Saline solution $(0.9 \%)$ and Minoxyl ${ }^{\mathbb{R}}$ (minoxidil $5 \%$ solution) were also used as negative and positive control, respectively. The degree of hair growth was evaluated by visual scoring of hair growth quantification scale as re-

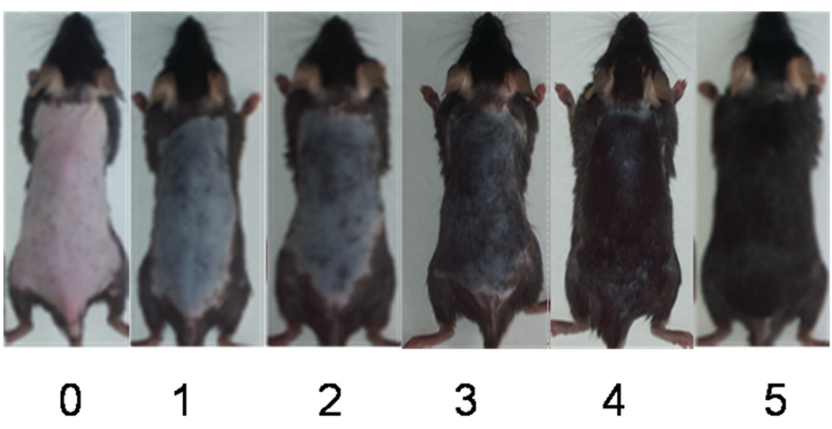

Fig. 2. Hair growth quantification scale. 0 : initial state, 1: grey to black coloration, 2: short visible hair, 3: sparse long hair, 4: dense long hair, 5: complete hair growth.

ported by Chen and his colleagues ( 0 : initial state, 1 : grey to black coloration, 2: short visible hair, 3: sparse long hair, 4: dense long hair, 5: complete hair growth) (Fig. 2) (Chen et al., 2005). Photographs of all mice in this study were taken every five days for 20 days.

\section{Transdermal penetration and intradermal retention studies}

Hairless mice were sacrificed and full-thickness skin was taken, followed by removal of subcutaneous fat. Approximately $3 \mathrm{~cm}^{2}$ of the trimmed skin was placed into Franz diffusion cell with effective diffusion area of 2.27 $\mathrm{cm}^{2}$. For transdermal penetration study, freshly prepared aqueous solution $(0.4 \mathrm{ml})$ of MAP $7.5 \%$ alone or with MSM $1 \%, 5 \%$ or $10 \%$ was applied on the skin and allowed to spread over the skin. Donor compartment (epidermal layer of the skin) remained unoccluded, but covered with a lid, and the receptor compartment (dermal layer of the skin) was perfused with distilled water maintaining temperature at $37^{\circ} \mathrm{C}$. Serial sampling of the receptor compartment was performed at predetermined intervals $(1.5,3,6,9$, and 12 h). Cumulative amount of MAP penetrated through the skin was plotted as a function of time. The flux of MAP was calculated by the slope of the linear portion of the curve, which was achieved in $6 \mathrm{~h}$

For intradermal retention study, the effective diffusion area of the mounted skin was cut off after transdermal penetration study and washed with distilled water, and stored in the deep-freezer until HPLC determination. For analysis, the skin was homogenized with isotonic phosphate buffer (pH 7.4) for $10 \mathrm{~min}$ in an ice bath. To $0.45 \mathrm{ml}$ of the skin homogenate, $0.05 \mathrm{ml}$ of internal standard $(100 \mu \mathrm{g} / \mathrm{ml})$ solution and $0.5 \mathrm{ml}$ of extraction solvent (10\% metaphosphoric acid:20\% trichloroacetic acid:distilled water=2:4:4) were added, and was subjected to sonication for $10 \mathrm{~min}$ and centrifugation at $16,000 \mathrm{~g}$ for $3 \mathrm{~min}$. The clear super- 


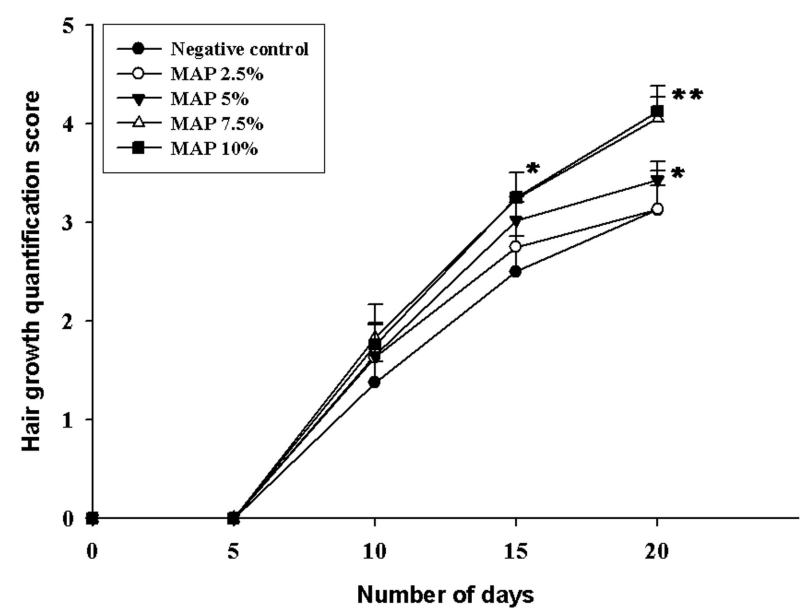

Fig. 3. Hair growth quantification score after application of MAP in alopecia model of C57BL/6N mice $(n=5)$. MAP: magnesium ascorbyl phosphate, * and ${ }^{* *}$ represent $p<0.05$ and $<0.01 \mathrm{com}$ pared to negative control, respectively.

natant thus obtained was then analyzed for MAP by HPLC utilizing the method described in HPLC assay section.

\section{Data analysis}

All the data obtained were analyzed using SPSS 12.0 for Windows and a significance level of $p<0.05$ was used to indicate the statistically significant difference between data sets.

\section{RESULTS}

Aqueous solution $(0.2 \mathrm{ml})$ of MAP was investigated for the ability to promote hair growth in the alopecia model of C57BL/6N mice. There was no significant difference between the MAP-treated groups and control group up to $10^{\text {th }}$ day (Fig. 3). However, in $15^{\text {th }}$ day, hair growth quantification score in the mice treated with MAP $7.5 \%$ or above was significantly enhanced compared to control group ( $p$ $<0.05$ ), and the hair growth status of the mice was "dense long hair" in $20^{\text {th }}$ day. MAP $5 \%$ also showed hair growth promotion compared to control group in $20^{\text {th }}$ day $(p<0.05)$, but hair growth status of the mice was in the level of "sparse long hair". Hair growth status in the group treated with MAP $2.5 \%$ was not different from control group even in $20^{\text {th }}$ day.

The effect of MSM on the hair growth promotion of MAP $7.5 \%$ solution was investigated in the presence of MSM $1 \%, 5 \%$ or $10 \%$ (Fig. 4). MSM $1 \%$ did not result in significant additive effect even after 20 days. However, MSM $10 \%$ demonstrated statistically significant additive effect as early as in $10^{\text {th }}$ day $(p<0.05)$, showing $2.50 \pm 0.33$ of

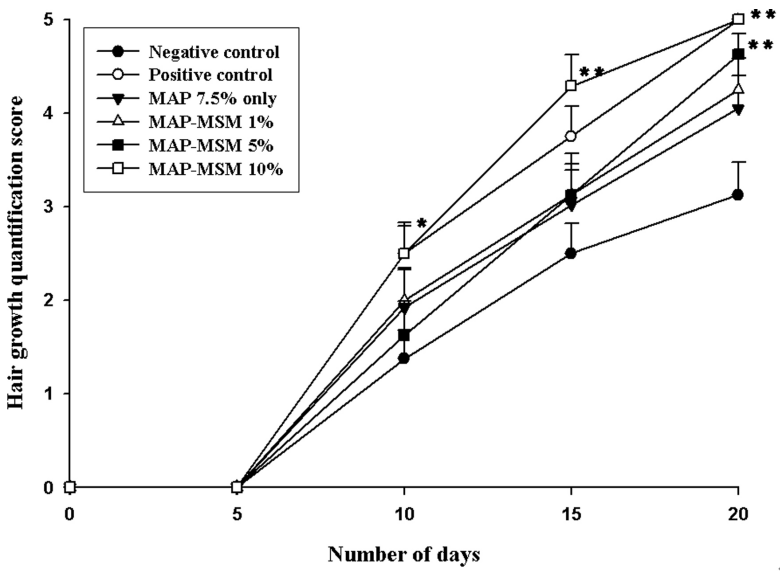

Fig. 4. Hair growth quantification score after application of MAP $7.5 \%$ with or without MSM in alopecia model of C57BL/6N mice $(n=5)$. MAP: magnesium ascorbyl phosphate, MSM: methylsulfonylmethane, MAP-MSM: MAP $7.5 \%$ in the presence of designated concentration of MSM, * and ** represent $p<0.05$ and $<0.01$ compared to MAP $7.5 \%$ only, respectively.

hair growth quantification score compared to $1.92 \pm 0.33$ in the group treated with MAP $7.5 \%$ solution without MSM. After 20 days of treatment, hair growth status in the group treated with MAP $7.5 \%$ solution with MSM $5 \%$ or $10 \%$ was "complete hair growth", while it was still in the level of "dense long hair" in the group treated with MAP $7.5 \%$ solution without MSM $(p<0.01)$. Furthermore, hair growth rate in the mice treated with MAP $7.5 \%$ solution with MSM $10 \%$ was better than or similar to the mice treated with minoxidil $5 \%$ solution. Photographs for hair growth status in all groups tested are shown in Fig. 5.

Cumulative amount of MAP penetrated through the hairless mice skin during $12 \mathrm{~h}$ was $3.89 \pm 0.41,2.23 \pm 0.39$, and $1.80 \pm 0.24 \mu \mathrm{g} / \mathrm{cm}^{2}$ in the presence of MSM $1 \%, 5 \%$, and $10 \%$, respectively, while it was $4.17 \pm 0.46 \mu \mathrm{g} / \mathrm{cm}^{2}$ in the absence of MSM (Fig. 6). Lag time for transdermal penetration of MAP was about $1.5 \mathrm{~h}$, and MAP was detected in the receptor compartment of the Franz diffusion cell after $3 \mathrm{~h}$. When MAP was applied as $7.5 \%$ solution with $5 \%$ or $10 \%$ MSM, transdermal penetration of MAP was only $53.5 \%$ and $43.2 \%$ of the amount penetrated in the absence of MSM, respectively $(p<0.01)$. Average flux of MAP calculated by the slope of the linear portion of each curve was $0.08080 \pm .04,0.0359 \pm 0.01$, and $0.0313 \pm 0.02 \mu \mathrm{g} \times \mathrm{cm}^{-2} / \mathrm{h}$ in the presence of MSM $1 \%, 5 \%$, and $10 \%$, respectively, while it was $0.2205 \pm 0.02 \mu \mathrm{g} \times \mathrm{cm}^{-2} / \mathrm{h}$ in the absence of MSM (Table II). The flux of MAP in the presence of MSM $5 \%$ or above was less than one fifth of the flux in the absence of MSM.

The amount of MAP remaining in the skin (intradermal 


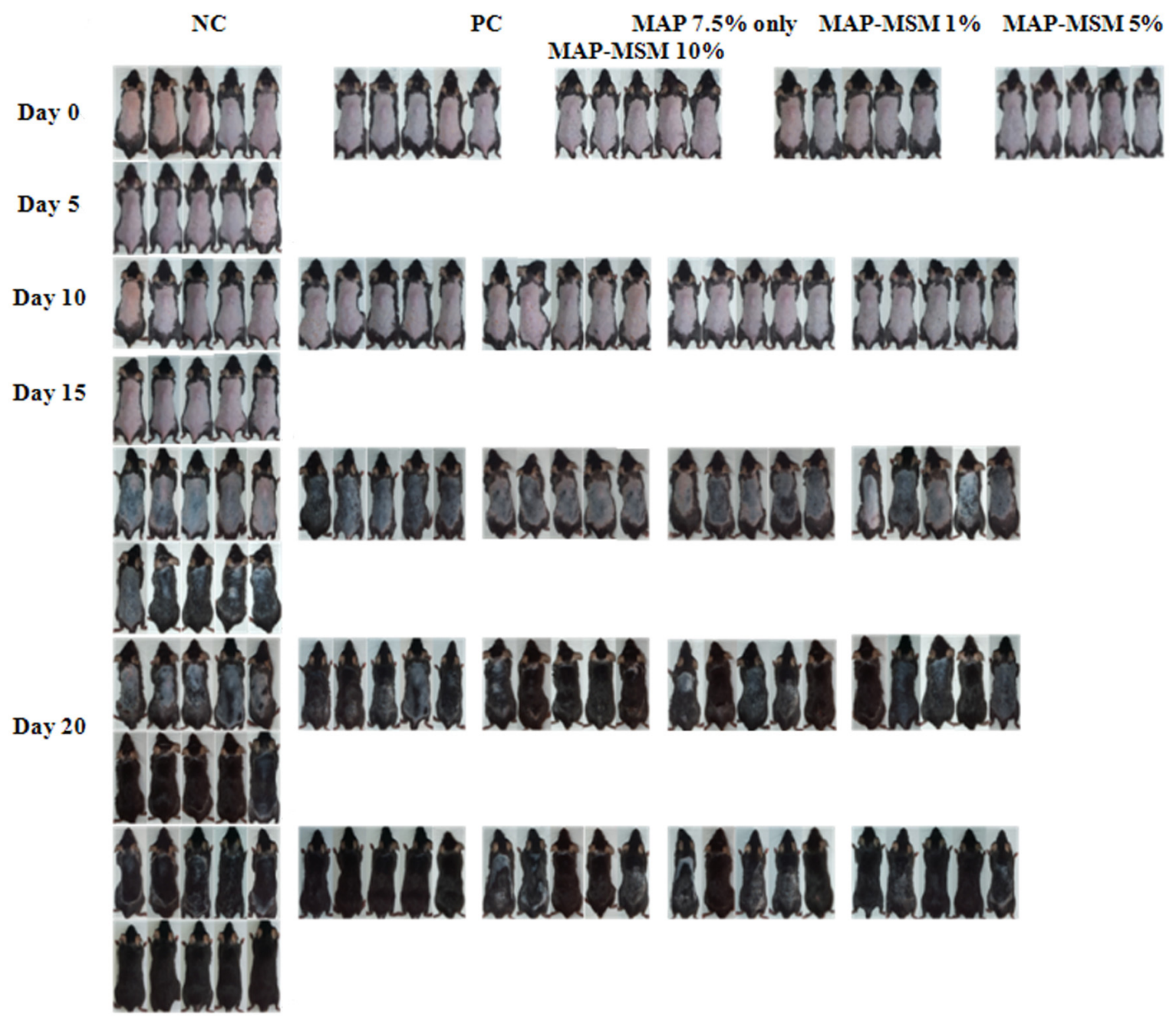

Fig. 5. Photographs of hair growth status during treatment with MAP in the absence or presence of MSM. MAP: magnesium ascorbyl phosphate, MSM: methylsulfonylmethane, MAP-MSM: MAP $7.5 \%$ in the presence of designated concentration of MSM, NC: negative control ( $0.9 \%$ saline solution), PC: positive control (minoxidil $5 \%$ solution).

retention) after $12 \mathrm{~h}$ of transdermal penetration study was $10.5 \pm 12.1,62.0 \pm 34.6$, and $801.7 \pm 156.0 \mu \mathrm{g} / \mathrm{cm}^{2}$ in the presence of MSM $1 \%, 5 \%$, and $10 \%$, respectively. It was only $4.2 \pm 5.9 \mu \mathrm{g} / \mathrm{cm}^{2}$ when MAP was applied without MSM (Fig. 7). Intradermal retention of MAP was exponentially increased in the presence of MSM, reaching about 200-fold when MAP was applied with MSM $10 \%$.

\section{DISCUSSION}

Hair growth in animals periodically undergoes a continuous hair cycle, during which the hair follicle switches between phases of growth and regression (Paus, 2006). During the hair cycle, the growth phase (anagen) lasts for two to seven years followed by transition and involution phase (catagen), which lasts for one to two weeks, and fi- nally the resting phase (telogen) that lasts for five to 12 weeks (Ellis et al., 2002). MAP was reported to enhance the hair growth through anagen induction, which is considered one of the most effective strategies for treating hair loss (Paus, 2006).

As a preliminary study, we investigated hair growth promotion effect of MAP in alopecia model using black-haired mice (C57BL/6N). Concentrations of MAP tested in this study were $2.5 \%, 5 \%, 7.5 \%$, and $10 \%$, because previous researchers reported that MAP induced early conversion from telogen phase to anagen phase of hair growth cycle at $250 \mathrm{mM}$ concentration, which is equivalent to about $7.25 \%$ of MAP (Sung et al., 2006). Hair growth quantification score in the groups treated with MAP was in proportion to the concentration of MAP up to $7.5 \%$ and plateaued above this concentration. This result is consistent with the 
Table II. Average flux of MAP in the skin penetration study $(n=6)$

\begin{tabular}{ccccc}
\hline & MAP 7.5\% only & MAP-MSM 1\% & MAP-MSM 5\% & MAP-MSM 10\% \\
\hline Average flux $\left(\mu \mathrm{g} \times \mathrm{cm}^{-2} / \mathrm{h}\right)$ & $0.2205 \pm 0.02$ & $0.0808 \pm 0.04^{\mathrm{a}}$ & $0.0359 \pm 0.01^{\mathrm{a}}$ & $0.0313 \pm 0.02^{\mathrm{a}}$ \\
\hline
\end{tabular}

MAP: magnesium ascorbyl phosphate, MSM: methylsulfonylemethane, MAP-MSM: MAP 7.5\% in the presence of designated concentration of MSM, ${ }^{a} p<0.01$ compared to MAP $7.5 \%$ only.

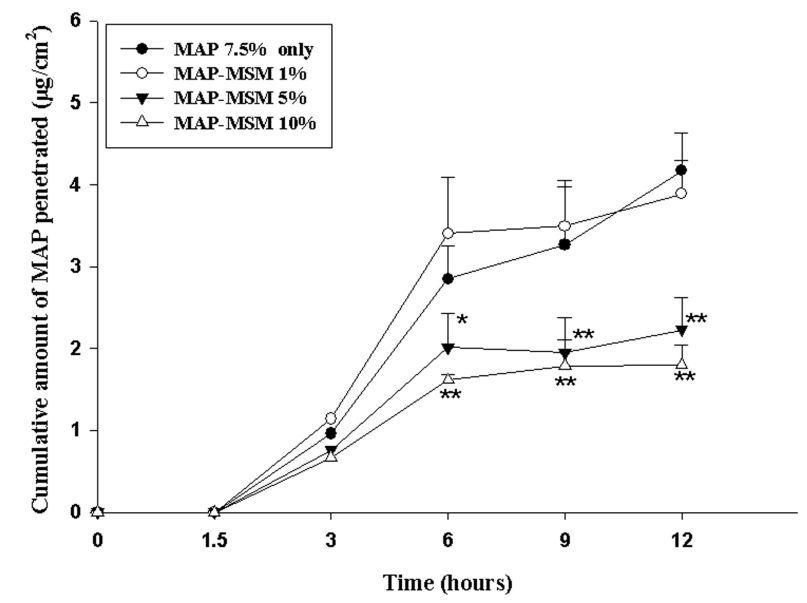

Fig. 6. Amount of MAP penetrated through hairless mice skin $(n=5)$. MAP: magnesium ascorbyl phosphate, MSM: methylsulfonylmethane, MAP-MSM: MAP $7.5 \%$ in the presence of designated concentration of MSM, * and ** represent $p<0.05$ and $<0.01$ compared to MAP $7.5 \%$ only, respectively.

previous researchers' finding that MAP promotes hair growth (Sung et al., 2006).

Hair growth status in the group treated with combination of MAP $7.5 \%$ solution and MSM $5 \%$ or $10 \%$ was significantly better than the result found in the group treated with MAP $7.5 \%$ solution only $(p<0.01)$. Notably, hair growth in the group treated with MAP $7.5 \%$ solution and MSM $10 \%$ showed better than or similar to the group treated with minoxidil $5 \%$ solution (positive control), which is considered a standard topical therapy for the treatment of alopecia. Also remarkably, the hair growth status was in proportion to the concentration of MSM, suggesting that MSM played a role as a hair growth promotion agent. Due to its high sulfur content, MSM has been believed to nourish skin, hair, and finger nails (Richmond, 1986). So, we performed a preliminary experiment to see if MSM itself has hair growth promotion effect in the concentration range of $1-10 \%$ using mice alopecia model. The experiment, however, did not show any hair growth promotion effect of MSM in the concentration range tested. These findings ruled out the probable hair growth ability of MSM and demonstrated that the observed hair growth promotion in MAP and MSM treated mice was only due to MAP. However, the dose-dependent enhancement in hair

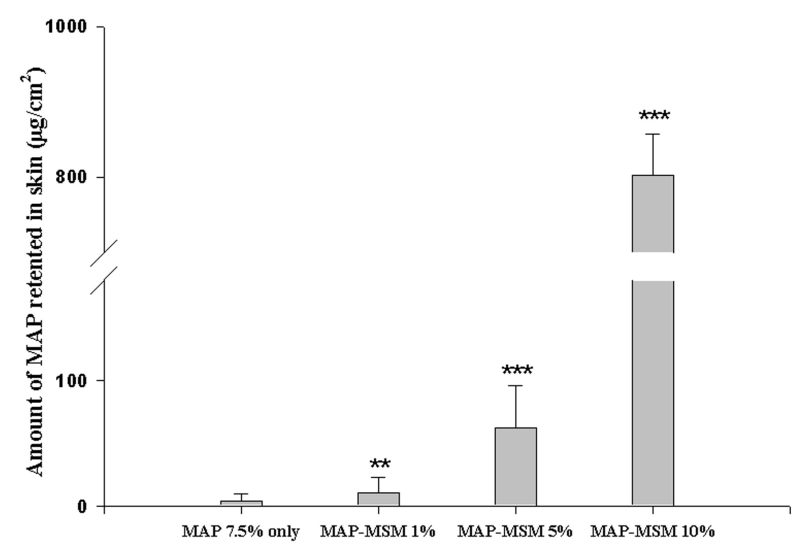

Fig. 7. Amount of MAP accumulated in hairless mice skin $(n=5)$. MAP: magnesium ascorbyl phosphate, MSM: methylsulfonylmethane, MAP-MSM: MAP $7.5 \%$ in the presence of designated concentration of MSM, ${ }^{* *}$ and ${ }^{* * *}$ represent $p<0.01$ and $<0.001$ compared to MAP $7.5 \%$ only, respectively.

growth with increasing MSM concentration implied significant role of MSM as a penetration enhancer, which could have exerted its additive effect on the hair growth of MAP $7.5 \%$ solution by enhancing transdermal delivery of MAP to hair follicles in the dermis layer of the skin.

In the present study, based on the speculation above (enhanced transdermal delivery of MAP with the aid of MSM), we performed transdermal penetration study of MAP $7.5 \%$ solution in the presence of $\operatorname{MSM}(1,5$, and $10 \%)$ using Franz diffusion cell. We expected that transdermal penetration of MAP would be significantly increased in the presence of MSM, because MSM is structurally very similar to dimethyl sulfoxide, a very strong skin permeation enhancer (van Engelen and van Engelen, 2002). Unexpectedly, in this experiment, the transdermal penetration of MAP was significantly decreased when MAP was applied with MSM concentrations of $5 \%$ or above (Fig. 5) compared to control. This unexpected result led us take into consideration that transdermal permeation enhancers might be subdivided into several classes. In fact, Asbill and his colleagues have categorized the enhancers into those that enhance transdermal penetration across the skin, those enhance intradermal retention into the skin, and those enhance both (Asbill and Michniak, 2000). In fact, accumulation of drug within skin without sig- 
nificant systemic appearance is the objective of any means of topical delivery (Trommer and Neubert, 2006).

Accordingly, as an attempt to substantiate the possibility of accumulation of MAP within the skin by MSM, we measured the amount of MAP remaining in the skin (intradermal retention) after $12 \mathrm{~h}$ of transdermal penetration study. Surprisingly, we found that the amount of MAP in the skin was profoundly and dose-proportionally increased as a function of MSM concentration. The increase was almost about 200 -fold in the presence of MSM $10 \%$, compared to the result in the absence of MSM (Fig. 7). Though, the exact mechanism by which MSM enhances intradermal permeation of MAP is unclear now, MSM might have followed the similar mechanism of DMSO due to the structural similarity, principally with the possession of characteristic polar O-S bond, which enables the compound to interact strongly with water by forming hydrogen bonds. The hydrogen-bonding ability of a substance is a major determinant of penetration. Therefore, the hydrogen-bonding groups of the enhancers could indeed modify the penetration (Abraham et al., 1995; Potts and Guy, 1995). Although DMSO is considered a strong permeation enhancer, its permeation enhancing effect is dose-dependent and in general, more than $60 \%$ DMSO is needed for optimal permeation enhancing effect. Nevertheless, at such high concentrations, DMSO is reported to cause erythema and wheals of the stratum corneum and may denature skin protein (Williams and Barry, 2003).

It is worth noting that MSM possesses two polar O-S groups (Fig. 1B) that could have efficiently bound to water of stratum corneum forming hydrogen bonds and aided penetration of the solutes. Probably, the same reason could be applicable for its penetration effectiveness even at low concentration of about $10 \%$ or less compared DMSO. It is possible that the high hydrogen bonding ability of MSM might have perturbed the stratum corneum sufficiently for the fast entry of MAP into the skin, while the low concentration (and subsequent reduction in concentration of MSM, due to its high penetration rate through the skin in to the systemic/receptor compartment) may not be sufficient to enhance the penetration of huge amount of MAP accumulated in the skin to penetrate through the skin. It is also possible that the strong interaction of MSM with water molecules might have resulted in less hydrophilic environment for MAP which makes its partitioning difficult leading to accumulation rather than further penetration (Kim et al., 1999). Also, it appears that skin accumulation effect of MSM was exponential to the concentration of MSM in the formula applied to the skin. However, without further mechanistic studies, we could not understand the exact mechanism by which MSM exerts its skin accumulation enhancing effect.

Despite the fact that the data reported do not directly reveal mechanistic information on percutaneous penetration and enhanced intradermal accumulation, they do provide a clear correlation of effect of MSM on the hair growth promotion effect of MAP in vivo and substantial intradermal accumulation of MAP in vitro, which was the primary objective of this study. Furthermore, this finding could be utilized for optimization and exploitation of intradermal drug delivery of MAP for the treatment of alopecia.

In conclusion, the results of the present study indicate that topical application of magnesium ascorbyl phosphate (MAP) and methylsulfonylmethane (MSM) is effective in promoting hair growth in C57BL/6N mice in vivo. Authors, first time to our knowledge report that, MSM could be used as a penetration enhancer that augments hair growth promotion of MAP in vivo through higher dermal accumulation of MAP demonstrated by in vitro skin study. The penetration enhancing effect of MSM was found to be dose-dependent, both in vivo and in vitro. Overall, topical application of MAP together with MSM appears to be useful for the treatment of alopecia.

\section{ACKNOWLEDGMENTS}

This work was supported by Korea Research Foundation Grant funded by the Korean Government (MOEHRD, Basic Research Promotion Fund) (KRF-2007-314-E00246).

\section{REFERENCES}

Abraham, M. H., Chadha, H. S. and Mitchell, R. C. (1995). The factors that influences skin penetration of solutes. J. Pharm. Pharmacol. 47, 8-16.

Ameye, L. G. and Chee, W. S. (2006). Osteoarthritis and nutrition. From nutraceuticals to functional foods: a systematic review of the scientific evidence. Arthritis Res. Ther. 8, R127.

Asbill, C. S. and Michniak, B. B. (2000). Percutaneous penetration enhancers: local versus transdermal activity. PSTT 3, 36-41.

Barrager, E., Veltmann Jr. J. R., Schauss, A. G. and Schiller, R. N. (2002). A multicentered, open-label trial on the safety and efficacy of methylsulfonylmethane in the treatment of seasonal allergic rhinitis. J. Altern. Complement. Med. 8, 167173.

Blum, J. M. and Blum, R. I. (2004). The effect of methylsulfonylmethane (MSM) in the control of snoring. Integrative Medicine 3, 24-30.

Chen, C. H., Sheu, M. T., Wu, A. B., Lin, K. P. and Ho, H .O. (2005). Simultaneous effects of tocopheryl polyethylene glycol succinate (TPGS) on local hair growth promotion and systemic absorption of topically applied minoxidil in a mouse 
model. Int. J. Pharm. 306, 91-98.

Childs, S. J. (1994). Dimethyl sulfone (DMSO2) in the treatment of interstitial cystitis. Urol. Clin. North Am. 21, 85-88.

Clark, T., Murray, J. S. and Lane, P. (2008). Why are dimethyl sulfoxide and dimethyl sulfone such good solvents? J. Mol. Model 14, 689-697.

Ellis, J. A., Sinclair, R. and Harrap, S. B. (2002). Androgenetic alopecia: pathogenesis and potential for therapy. Expert Rev. Mol. Med. 4, 1-11.

Engelen, V. H. W. and Engelen, V. P. A. (2002). Topical dermal anaesthetic. US Patent 6,416,772B1.

Franz, T. J., Lehman, P. A. and Kagy, M. K. (1995). Dimethyl sulfoxide. In Percutaneous Penetration Enhancers (E. W. Smith and H. I. Maibach, Eds.), pp.115-127. CRC Press, Boca Raton.

Godwin, D. A. and Michniak, B. B. (1999). Influence of drug lipophilicity on terpenes as transdermal penetration enhancers. Drug Dev. Ind. Pharm. 25, 905-915.

Hata, R. and Senoo, H. (1989). L-ascorbic acid 2-phosphate stimulates collagen accumulation, cell proliferation, and formation of a three-dimensional tissuelike substance by skin fibroblasts. J. Cell Physiol. 138, 8-16.

Im, T. J., Kang, M. J., Seo, D. W. and Lee, J. (2008). Effect of cubic liquid crystalline systems on skin localization of oregonin and hirsutanonol. Biomolecules \& Therapeutics. 16, 226-230.

Kim, L. S., Axelrod, L. J., Howard, P., Buratovich, N. and Waters, R. F. (2006). Efficacy of methylsulfonylmethane (MSM) in osteoarthritis pain of the knee: a pilot clinical trial. Osteoarthritis Cartilage 14, 286-294.

Kim, N., El-Khalili, M., Henary, M. M., Strekowski, L. and Michniak, B. B. (1999). Percutaneous penetration enhancement activity of aromatic S, S-dimethyliminosulfuranes. Int. J. Pharm. 187, 219-229.

Paus, R. (2006). Therapeutic strategies for treating hair loss. Drug Discov. Today Ther. Strateg. 3, 101-110.

Pearson, T. W., Dawson, H. J. and Lackey, H. B. (1981) Natural occurring levels of dimethyl sulfoxide in selected fruits, vegetables, grains, and beverages. J. Agric. Food. Chem. 29, 1089-1091.
Potts, R. O. and Guy, R. H. (1995). A predictive algorithm for skin permeability: the effects of molecular size and hydrogen bond activity. Pharm. Res. 12, 1628-1633.

Richmond, V. L. (1986). Incorporation of methylsulfonylmethane sulfur into guinea pig serum proteins. Life Sciences 39, 263-268.

Skelly, J., Shah, V., Maibach, H., Guy, R., Flynn, G. and Yacobi, A. (1987). FDA and AAPS report of the workshop on principles and practices of in vitro percutaneous penetration studies: relevance to bioavailability and bioequivalence. Pharm. Res. 4, 265-267.

Spiclin, P., Homar, M., Zupancic-Valant, A. and Gasperlin, M. (2002). Sodium ascorbyl phosphate in topical microemulsions. Int. J. Pharm. 256, 65-73.

Sung, Y. K., Hwang, S. Y., Cha, S. Y., Kim, S. R., Park, S. Y., Kim, M. K. and Kim, J. C. (2006). The hair growth promoting effect of ascorbic acid 2-phosphate, a long-acting vitamin C derivative. J. Dermatol. Sci. 41, 150-152.

Takamizawa, S., Maehata, Y., Imai, K., Senoo, H., Sato, S. and Hata, R. (2004). Effects of ascorbic acid and ascorbic acid 2-phosphate, a long-acting vitamin $\mathrm{C}$ derivative, on the proliferation and differentiation of human osteoblast-like cells. Cell Biol. Int. 28, 255-265.

Trommer, H. and Neubert, R. H. (2006). Overcoming the stratum corneum: the modulation of skin penetration. Skin Pharmacol. Physiol. 19, 106-121.

Usha, P. R. and Naidu, M. U. (2004). Randomised, double-blind, parallel, placebo-controlled study of oral gGlucosamine, methylsulfonylmethane and their combination in osteoarthritis. Clin. Drug Investig. 24, 353-363.

Williams, A. C. and Barry, B. W. (1992). Skin absorption enhancers. Crit. Rev. Ther. Drug Carrier Syst. 9, 305-353.

Williams, A. C. and Barry, W. B. (2003). Penetration enhancers. Adv. Drug Deliv. Rev. 56, 603-618.

Yoo, J., Shanmugam, S., Song, C. K., Kim, D. D., Choi, H. G., Yong, C. S., Woo, J. S. and Yoo, B. K. (2008). Skin penetration and retention of L-ascorbic acid 2-phosphate using multilamellar vesicles. Arch. Pharm. Res. 31, 16521658. 